\title{
ANÁLISIS DE LA PRODUCCIÓN Y TRADICIÓN CERAMISTA EN EL BARRIO DE ARTESANOS ICHIMAY WARI, LURÍN, PERÚ
}

\author{
ANALYSIS OF THE PRODUCTION AND CERAMIC TRADITION IN THE \\ ICHIMAY WARI ARTISAN NEIGHBORHOOD, LURIN, PERU
}

\author{
Milenka Cáceres Hurtado \\ Universidad de San Martín de Porres, Perú \\ Elizabeth Sara Gomez Castillo \\ Universidad de San Martín de Porres, Perú
}

Aceptado: 15 de febrero de 2020

\section{RESUMEN}

Se analiza y describe la realidad de la tradición ceramista en Ichimay Wari, Lurín, Perú, con el objetivo de proponer acciones que permitan salvaguardar su tradición ceramista; y teniendo como base la investigación Análisis del impacto de la producción masiva en la tradición ceramista de la comunidad Ichimay Wari, Lurín, 2018 (Cáceres, 2019). Los resultados permiten afirmar la importancia de mantener la tradición ceramista pues es parte del patrimonio cultural inmaterial y a la vez es importante como símbolo de identidad cultural. Ya que la cerámica es un producto cultural, es necesario considerar ciertos aspectos que permitan la comercialización, dándole el valor que merece en el mercado sin dejar de lado la importancia en cuanto a identidad que tiene cada una de las piezas para el artesano.

Palabras clave: artesanía, tradición ceramista, conservación, patrimonio inmaterial.

\begin{abstract}
The pottery tradition in Ichimay Wari, Lurín, Peru is analyzed and described, with the aim of proposing actions that allow its safeguarding; based on the research «Analysis of the impact of mass production on the ceramic tradition of the Ichimay Wari community, Lurín, 2018» (Cáceres, 2019). The results confirm the importance of maintaining the ceramic tradition as it is part of the intangible cultural heritage and at the same time, it is important as a symbol of cultural identity. Since ceramics is a cultural product, it is necessary to consider certain aspects that allow its commercialization, giving it the value it deserves in the market without neglecting its identity value and the importance of each of the pieces for the artisan.
\end{abstract}

Keywords: crafts, ceramic tradition, conservation, intangible heritage.

Este es un artículo Open Access bajo la licencia Creative Commons AtribuciónNoComercial-Compartirlgual 4.0

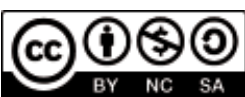




\section{Introducción}

En el distrito de Lurín, al sur de Lima capital del Perú, se ubica la Asociación de Artesanos Ichimay Wari, integrada por migrantes provenientes de Ayacucho. Gran número de estos artesanos se desplazaron de su lugar de origen por diversos factores, vieron en la capital la oportunidad de desarrollarse dentro del campo artesanal, al haber aprendido de sus ancestros el arte, la tradición y las diversas técnicas artesanales.

Los saberes adquiridos, además de la destreza de sus manos, se considera que es debido al aporte de diversas culturas que se desarrollaron en Ayacucho, entre ellos la Cultura Wari a cuyos pobladores se les reconoce en la historia como guerreros y hábiles artesanos (Zapata, Rojas y Pereyra, 2013). La influencia de estas dos culturas prehispánicas así como la manifestación del cristianismo, traído por los españoles, se ven representadas en la elaboración de sus cerámicas.

Respecto a su organización, fue durante el año 1999 cuando estos artesanos, ya asentados en el valle de Lurín, decidieron organizarse como asociación formal. Posteriormente en el año 2001 se constituyeron legalmente con el nombre Ichimay Wari, cuyo origen proviene de dos vocablos. El primero Ichimay, que se deriva de Ichma nombre de una cultura que se desarrolló en la Región Lima, de forma autónoma «aproximadamente en el año 900 hasta el 1450 de nuestra era, y sujetos al Tahuantinsuyo desde entonces hasta 1535» (Espinoza, s. f.); y el segundo, Wari que hace referencia a la cultura que surgió alrededor de 550-600 d. C. en Ayacucho, según el arqueólogo Luis G. Lumbreras (Bueno, 2003). A raíz de este mestizaje cultural nace una nueva propuesta de arte cerámico y con ella el denominado «barrio de artesanos» de Lurín, en Lima.

En la actualidad, los Ichimay Wari se dedican a la producción masiva de cerámica utilitaria, decorativa y tradicional; siendo esta última, elaborada en un menor porcentaje pues su proceso de producción se ha visto influenciada por elementos contemporáneos que han modificado el diseño, la forma y la materia prima con los que se elaboran. Esta realidad, de acuerdo a los pobladores entrevistados, es a consecuencia de la disminución de las ventas de cerámicas tradicionales, problema que a su vez ha generado la reducción de los ingresos económicos, que obtenían al dedicarse exclusivamente a esta actividad.

Frente a este escenario, los artesanos buscaron incrementar su comercialización optando como pronta solución encajar en el competitivo mercado internacional de artesanías, dejando así de lado esas características tradicionales, fruto del saber adquirido, que expresaban su identidad. Asimismo, esta situación ha generado la pérdida de interés de las siguientes generaciones, quienes han optado por dedicarse a otras actividades poniendo en riesgo la continuidad de la producción y a su vez la desaparición de esta actividad tradición artesanal, patrimonio cultural y con ello aquel legado cultural que se ha trasmitido de generación en generación.

El objetivo del presente estudio es analizar las causas de la disminución en la elaboración de la cerámica tradicional en la asociación Ichimay Wari, para así poder proponer la preservación del patrimonio cultural inmaterial. En el desarrollo de la investigación se recurrió al análisis de casos, uno de ellos es el estudio realizado por la Dirección General de Política de la pequeña y mediana empresa del Ministerio de Industria, Turismo y Comercio de España, realizado en el año 2011. Este estudio tuvo como objetivo elaborar un diagnóstico de la situación de la industria artesanal, mediante encuestas aplicadas a empresas dedicadas a este oficio. 
En el estudio se encontró que los artesanos de las comunidades de Cataluña, Andalucía, Valencia, Galicia y Castilla de la Mancha aún conservan la trayectoria familiar debido a que, en su entorno territorial, la cerámica es una de las principales actividades que se desarrollan, siendo su fuente básica de ingreso. Sin embargo, las entrevistas en profundidad que se efectuaron, permitieron identificar la presencia de una creciente pérdida de las tradiciones artesanales, particularmente en el caso de los artesanos de Cataluña, Valencia y Madrid; preocupando así el futuro de los mismos.

Por otro lado, con relación a las comunidades andinas, la investigación cualitativa realizada a la comunidad Huancarucma en Ayacucho, desarrollada por Hermoza (2010), tuvo como objetivo identificar las dinámicas sociales presentes en esta comunidad mediante talleres participativos, donde se realizaron entrevistas, a los que el autor llama, «actores externos»; es así que demostró lo debilitada que se encuentra esta comunidad respecto a sus tradiciones. De esta forma logra reflexionar en la importancia de la valoración, identificación y rescate de las técnicas ancestrales que permitan fortalecer su identidad con el fin de incentivarla, y como consecuencia, lograr su preservación.

Si bien el tema de resguardar la tradición en la elaboración de los productos es transcendental como parte de la historia de una cultura, también es importante reconocer que a lo largo del tiempo los procesos suelen adquirir nuevas características que resultan a veces necesarias para poder mantenerlos en vigencia, ya que aportan creatividad e innovación al producto. Sin embargo, se considera fundamental que estas características y procesos puedan también contribuir con el rescate de técnicas y procesos ancestrales.

Es así que después de haber analizado los diferentes enfoques relacionados con la producción artesanal tradicional, se comprobó que es necesario identificar y analizar los cambios que surgen en la tradición ceramista de la comunidad Ichimay Wari a partir de la producción masiva, para luego proceder en el registro de las características propias de la tradición artesanal, como el estilo, diseño, iconografías, materias primas, entre otros aspectos que la distinguen de las otras comunidades artesanas.

El resultado del análisis de estas particularidades, permitirán la elaboración de acciones que promuevan continuar con la producción artesanal, sin dejar de lado los saberes y técnicas que brinda la tradición ancestral que, en estos tiempos de globalización, es importante preservar como símbolo de identidad y patrimonio de una cultural local.

\section{La artesanía como patrimonio cultural inmaterial}

La artesanía desde la existencia del hombre representó diversos elementos, entre ellos su relación con la naturaleza, contexto social, cultura e historia. Su producción nace de un ambiente habitual y de trabajo, permitiéndoles así la creación de objetos de uso doméstico que facilitan el trabajo diario como el trasladar, contener y/o almacenar diversos insumos necesarios para la subsistencia. No obstante, más allá de mostrar su empleo utilitario, la artesanía tradicional también mostró funciones de connotación ritual. En el Perú, de acuerdo al Ministerio de Comercio Exterior y Turismo (MINCETUR): 
La artesanía peruana es una labor milenaria y de connotaciones mágicas. Cada pieza artesanal, elaborada en talleres a lo largo del país, es resultado de un saber transmitido entre artesanos, de padres a hijos, de generación en generación desde tiempos prehispánicos. A su vez, esa herencia ancestral es enriquecida perennemente por la imaginación del artesano, su relación con otras culturas y el empleo de nuevos instrumentos en su labor. (s. f., p. 10)

Por ello, es de suma importancia reconocer que las cerámicas artesanales son parte de la identidad y cultura; pues este patrimonio muestra el pasado, el presente y el futuro de una sociedad, lo cual permite ahondar en la razón por la cual deberían ser conservadas.

Respecto a la definición de patrimonio cultural inmaterial, la Organización de las Naciones Unidas para la Educación, la Ciencia y la Cultura (UNESCO), en la Convención del Patrimonio Cultural Inmaterial 2003, en el artículo 2 señala:

Los usos, representaciones, expresiones, conocimientos y técnicas junto con los instrumentos, artefactos y espacios culturales que les son inherentes, que las comunidades, los grupos y en algunos casos individuos reconozcan como parte de su patrimonio cultural. Este patrimonio cultural inmaterial, que se transmite de generación en generación, es recreado constantemente por las comunidades y grupos en función de su entorno, su interacción con la naturaleza y su historia, infundiéndoles un sentimiento de identidad y continuidad y contribuyendo así a promover el respeto de la diversidad cultural y la creatividad humana. (párr.16)

En este aspecto, la tradición ceramista reúne las características del concepto dado por la UNESCO pues es una expresión que se da a través del uso de artefactos, aquellas herramientas que requiere el autor para la creación de la cerámica; y además de técnicas, que son las expresiones de un colectivo quienes ven en esta actividad un elemento transmisor de vivencias, costumbres, modos de vida e incluso hechos históricos; aportando de esta forma particularidad a cada pieza elaborada, siendo así un objeto único.

De esta forma, estas piezas artesanales, logran ser recreadas constantemente por los miembros de las comunidades, consideradas como significativas por tener rasgos que los identifica, los diferencian y representan frente a los demás. «La artesanía, como producto folklórico, ha conformado rasgos distintivos de nuestra identidad, como individuos y como colectivo. Ese proceso ha estado determinado por el medio ambiente y la realidad cultural, social y económica» (Navarro, 2015, p. 3).

La razón de estos cambios es la interacción cultural que se da generacionalmente, es así que, a partir de estas características se suele generar la búsqueda de su preservación o salvaguardia, la cual promueve el respeto a la creatividad del hombre además de la diversidad cultural.

Con respecto a las técnicas artesanales UNESCO (s. f.) señala:

Son expresiones, que se transmiten de generación en generación; estas son muy numerosas y pueden ser representadas a través de joyas, indumentaria, prendas, accesorios, recipientes, objetos decorativos 
inclusive rituales, instrumentos musicales o enseres domésticos, elementos lúdicos, algunos de uso efímero al ser creados en un tiempo determinado para actividades específicas, u otros que constituyen un elemento heredado por el valor brindado a esa pieza. A su vez estos pueden ser objetos muy delicados, de acabado minucioso, o también pueden ser simples objetos con acabados más rústicos.

Todos estos saberes y prácticas que giran en torno a la producción de piezas artesanales, constituyen la cultura viva de un pueblo; siendo vulnerables frente a la falta de continuidad por factores internos y externos que se presentan en la transmisión y producción de este tesoro vivo, llegando así a poner en peligro su existencia.

Por ello, es importante salvaguardar aquellos saberes y prácticas que se vinculan con la artesanía tradicional, pues estas son la expresión de la cultura, la historia y la identidad de los pueblos, los cuales forman parte del patrimonio cultural inmaterial.

\section{La artesanía como símbolo de identidad}

Como se ha señalado, el valor que posee el trabajo artesanal por ser patrimonio cultural de los pueblos es invaluable. Por ello se debe desarrollar medidas que permitan salvaguardar los procesos y tradiciones en torno a la artesanía tradicional, con el fin de resguardar aquella expresión histórica, cultural e identitaria de las poblaciones.

El Perú llamado también país de todas las sangres, como bien lo denominó el escritor peruano José María Arguedas, hace referencia de las diversas manifestaciones culturales que coexisten en un mismo territorio. Esa particularidad y riqueza cultural, que lo distingue de otros, ha ido enriqueciéndose a través de los siglos gracias a la constante evolución que se expresa mediante diversos elementos que representan a toda una herencia cultural que puede ser mostrada en una pieza de cerámica.

Por lo tanto, el valor que el artesano le da a cada una de sus obras expresa su pertenencia cultural, lo que identifica su trabajo y el lugar donde se desarrolla; es así que el objeto artesanal es distinto a la producción industrial al tener como principal característica el estar elaborado a mano, ese distintivo está influenciado por el significado de acuerdo al contexto sociocultural en el que se elabora.

Asimismo, las prácticas tradicionales expresan las características de un grupo, es por ello común apreciar en algunos lugares del territorio nacional la cohesión generada a partir de la apropiación de sus costumbres.

\section{La cerámica artesanal y la sostenibilidad ambiental}

En la actualidad es necesario que los artesanos incluyan el tema de la sostenibilidad en los procesos de producción de las cerámicas artesanales, al ser de valiosa importancia el cuidado con el medio ambiente. De acuerdo a la tradición y cultura que guardan los antepasados de los Ichimay Wari, el respeto por la naturaleza es parte esencial dentro de los procesos de elaboración de las piezas artesanales. Sin embargo, una de las problemáticas actuales es que la artesanía al ser un producto cultural y comercial, se ha ido adaptando a las tendencias de la demanda masiva, siendo este su principal motivo de cambio, el cual ha llegado a vulnerar de manera significativa las técnicas y los procesos tradicionales de elaboración. 
Por ejemplo, el uso de las materias primas han variado y estas se han ido adaptando de acuerdo a la competencia del mercado. En el caso de la pintura, se ha dejado de usar pigmentos naturales para la decoración de las piezas artesanales; asimismo la producción masiva ha hecho que el artesano opte por simplificar sus técnicas en el forjado y el decorado, también ha crecido la tendencia a utilizar materias primas industrializadas, que muchas veces afectan el medio ambiente, todo ello con el fin de producir en menor tiempo y mejorar los costos de producción.

Teniendo en cuenta la Declaración de Río sobre el Medio Ambiente y el Desarrollo, aprobado en la Conferencia de las Naciones Unidas sobre el Medio Ambiente y el Desarrollo, se señala que «para alcanzar el desarrollo sostenible y una mejor calidad de vida para todas las personas, los Estados deberían reducir y eliminar las modalidades de producción y consumo insostenibles y fomentar políticas demográficas apropiadas» (Organización de las Naciones Unidas [ONU], 1992, p. 2); llama a la reflexión, como un aspecto que debe ser considerado tanto por el productor como el consumidor, con el fin de proteger al planeta. Si bien es cierto no se menciona a la artesanía de forma puntual, esto la involucra al ser una actividad productiva.

En ese sentido, es importante cumplir con las exigencias de sostenibilidad, considerando aplicar buenas prácticas de producción y operación de los procesos artesanales, como por ejemplo, el no agotar los recursos del entorno, en la selección del material a emplear, reducir la contaminación en la producción, fomentar el uso adecuado de insumos para elaborar las piezas, gestionar el uso de residuos generados e incluso reutilizar lo que se pueda; con el fin de que logre ser una actividad más responsable con el medio ambiente.

A propósito, en uno de los principios de la Declaración de Río sobre el Medio Ambiente y el Desarrollo se señala:

Las poblaciones indígenas y sus comunidades, así como otras comunidades locales, desempeñan un papel fundamental en la ordenación del medio ambiente y en el desarrollo debido a sus conocimientos y prácticas tradicionales. Los Estados deberían reconocer y apoyar debidamente su identidad, cultura e intereses y hacer posible su participación efectiva en el logro del desarrollo sostenible. (ONU, 1992, p. 5)

Cabe señalar así, la importancia de la recuperación y preservación de los saberes tradicionales relacionados con la cerámica artesanal, con el fin de resguardar el medio ambiente; encontrando así la armonía y el equilibrio entre el hombre y la naturaleza.

\section{Metodología}

Considerando las características de la investigación y que el tema recientemente es abordado, el enfoque metodológico que se empleó es el cualitativo. Para ello, se aplicó técnicas de observación, recopilación de datos así como entrevistas en profundidad, pues se tiene como propósito examinar la forma en cómo perciben o experimentan los individuos los fenómenos que los rodean; asimismo, se profundizó e interpretó sus puntos de vista. 
Con referencia al alcance, el estudio es descriptivo de las situaciones o eventos de forma precisa, asegurando de esta manera su comprensión. El diseño empleado es el fenomenológico ya que se estudia el hecho y se analizan los factores que contribuyen con la problemática del objeto de estudio. «Primero, se identifica el fenómeno y luego se recopilan datos de las personas que lo han experimentado, para finalmente desarrollar una descripción compartida de la esencia de la experiencia para todos los participantes -lo que vivenciaron y de qué forma lo hicieron» (Hernández, Fernández y Baptista, 2014, p. 493).

Para el desarrollo de la investigación, inicialmente se realizó el trabajo de campo, que permitió identificar el objeto de estudio a través de la observación. Posteriormente, mediante información obtenida al interactuar con la población, se pudo identificar la realidad y vivencias en los talleres artesanales de Ichimay Wari. De igual forma, con la guía de observación se logró registrar información relevante, para posteriormente elaborar y aplicar la entrevista a los artesanos.

Se utilizó como técnica la entrevista semiestructurada, que permite al entrevistador repreguntar de ser necesario, para poder enriquecer la información o precisar algunos conceptos. Para la elaboración de las preguntas se consideraron los siguientes aspectos: identidad cultural, cerámica tradicional y procesos de elaboración. De esta forma, se entrevistó a siete artesanos de la asociación objeto de estudio, dedicados a la actividad ceramista; seleccionados según criterio de conveniencia de la investigación, esto permitió que se obtuviera información de primera mano y comprender el punto de vista de los artesanos en relación con la problemática en análisis.

\section{Resultados}

la Asociación Ichimay Wari asociación estuvo integrada en un inicio por treinta artesanos. Con el paso del tiempo, por aspectos administrativos y otros motivos, varios de sus miembros optaron por ser independientes, es por ello que a la fecha la asociación cuenta con ocho artesanos, de los cuales, siete de ellos se dedican a la producción de cerámica artesanal.

En las entrevistas los artesanos manifestaron que sus costumbres están reflejadas en su cerámica, que la herencia cultural y la práctica constante les permiten mantener vigente sus tradiciones. Asimismo, señalaron que estas piezas artesanales son herencia de sus padres y expresan lo que los identifica como ayacuchanos.

En relación con la sostenibilidad de la tradición ceramista, los entrevistados reconocen que una de las formas de salvaguardar esta práctica es mediante la continuidad de la transmisión de los saberes que se dan de forma oral a través de las generaciones. Respecto a los aspectos de sostenibilidad medioambiental de su cerámica indicaron, que esta actividad no genera muchos residuos y que mientras la cerámica no está cocida puede reutilizarse el material que se emplea. Asimismo, señalan que gracias a las capacitaciones, que llevaron de forma particular, han aprendido a usar de manera responsable los insumos. Respecto a las pinturas que sirven para la decoración de las cerámicas, manifestaron que actualmente han dejado de lado el uso de pigmentos naturales y los han reemplazado por pigmentos sintéticos. 
Con relación a si existe un punto de equilibrio entre la cerámica tradicional y la moderna, la mayoría de entrevistados manifestaron que es difícil encontrar el equilibrio entre ambas. Al respecto, por ejemplo, el informante n. ${ }^{\circ} 2$ indicó que con el tiempo, la cerámica va perdiendo lo tradicional. Mientras que, para el informante $n .^{\circ} 4$, la cerámica tradicional tiene algunos aspectos que la han vuelto comercial. El informante $n .^{\circ} 6$ manifestó que aún se puede lograr que la cerámica tradicional no cambie en esencia.

Sobre la rentabilidad, los artesanos manifestaron que esta solo les permite cubrir sus necesidades básicas ya que actualmente ha disminuido mucho su venta en relación con años anteriores; así lo afirmó el informante n. ${ }^{\circ} 2$ quien comenta que las ventas han disminuido significativamente pero que a pesar de ello aún continúa dedicándose a esta actividad. Por su parte el informante.$^{\circ} 4$ señala que se dedica a la artesanía, para expresar sus costumbres y no por un tema económico. Por otro lado, el informante $n .^{\circ} 6$ manifestó que no es rentable los ingresos que ahora la artesanía le generan.

A la pregunta si aún se mantenían las tradiciones en el proceso de elaboración de sus piezas, coincidieron en que algunos de estos procesos han cambiado, pero que se trata de alguna forma de mantener las características esenciales heredadas de sus ancestros. El informante $n .{ }^{\circ} 2$ indicó que en los procesos, por ejemplo, se conserva muy poco lo tradicional. Hace referencia a que antes ellos mismos elaboraban el barro con el que trabajaban, ahora se compra este material. Por otro lado, el informante $n .^{\circ} 4$ indicó que con el tiempo todo ha cambiado y a su vez el informante $n .^{\circ} 6$ indicó que lo tradicional se conserva poco, siendo las características del diseño e iconografía las que aún se mantienen; al igual que las representaciones de elementos costumbristas, como técnica base.

Con referencia a los cambios que han realizado, manifestaron que debido a la exigencia de la demanda han optado por estilizar las formas de las cerámicas, para que así resulten piezas más atractivas; por ende, han cambiado los rasgos de los personajes andinos. El informante $n .^{\circ} 2$ indicó que al igual que las formas estilizadas también actualmente utiliza una gama de colores que no son los tradicionales. Por su parte, el informante n. ${ }^{\circ} 4$ indicó que su preocupación actual es estilizar las formas más no ha cambiado lo que representa; y el informante n. ${ }^{\circ} 6$ aseguró que tuvo que adaptarse a lo que el mercado solicita.

La demanda del mercado exige cambios en la artesanía. Por ello, con respecto a identificar las preferencias del mercado, los entrevistados indicaron que l mayoría solicitan objetos decorativos con colores llamativos, de acabado brillante y piezas pequeñas. El informante $n .{ }^{\circ} 2$ aseguró que sus piezas actualmente cumplen parte de estas características, con el objetivo de permanecer en el mercado. Por otro lado, el informante $n^{\circ} 4$ manifestó que, por ejemplo, en eventos importantes como el mundial de fútbol la demanda le exigía representar a los personajes de la selección peruana, es por ello que se dedicó a realizar estas piezas de cerámica. Por último el informante n. ${ }^{\circ} 6$ también indicó, al respecto, que la mayoría solicita piezas pequeñas y decorativas.

Con referencia a los aspectos que deberían considerarse para mantener la tradición Ichimay Wari, todos respondieron que el mantener la tradición, así como la preservación de los saberes ancestrales en torno a la artesanía, no solo depende de ellos, es necesario el apoyo de todos: la comunidad, las autoridades y entidades involucradas en esta actividad. Por su parte, el informante $n .^{\circ} 2$ afirmó que su responsabilidad es la transmisión 
familiar de estos saberes. El informante n. ${ }^{\circ} 4$ indicó que tal vez buscando nuevos nichos del mercado, que valoren su trabajo, podría subsistir en el tiempo; y finalmente el informante $n .^{\circ} 6$ indicó que es un trabajo en conjunto y coordinado entre las instituciones públicas y privadas.

\section{Conclusiones}

Mediante la investigación titulada: Análisis de la producción masiva en la tradición ceramista de la asociación Ichimay Wari Lurín, 2018, identificamos que la cerámica que poseen los artesanos de esta población aún conservan los rasgos andinos adquiridos de la unión de dos culturas, Ichma y Wari, como resultado de la transmisión de saberes de generación a generación. Asimismo, se considera que estas piezas artesanales poseen un carácter simbólico y cultural ya que los pobladores de este barrio de artesanos se sienten identificados y representados por las mismas. Es así que se destaca la importancia de mantener vigente las prácticas en torno a la cerámica artesanal.

Por otro lado, el estudio muestra los cambios que fueron generando en la producción de la cerámica artesanal, debido a las tendencias comerciales; uno de ellos es el uso de pigmentos sintéticos. Si bien, de acuerdo a los artesanos entrevistados, la pintura que ahora emplean les permiten tener una gran gama de colores, acabados más brillantes y rápido secado, esta contiene plomo, metal que puede ser perjudicial para la salud del artesano y su familia pues varios de los talleres artesanales se encuentran dentro de sus viviendas, exponiéndose así a la inhalación de este producto. El informe de la Cumbre Mundial sobre el Desarrollo Sostenible, realizado en Sudáfrica lo ratifica, pues señala:

[que se debe] eliminar gradualmente el plomo de las pinturas y de otras fuentes a que están expuestos los seres humanos; trabajar para prevenir, en particular, el contacto de los niños con el plomo y mejorar los esfuerzos de vigilancia y supervisión y el tratamiento del saturnismo. (ONU, 2002, p. 45)

Ante esta realidad, es recomendable retomar las prácticas y saberes ancestrales, considerándolo como característica trascendente, lo cual elevaría el valor del producto al ser sostenible y cuidadoso con el planeta.

Además, existe poca demanda de la cerámica tradicional siendo esta una problemática que pone en riesgo la producción; teniendo como consecuencia que esta actividad no sea rentable, y la falta de interés de las generaciones de artesanos que siguen. Por este motivo, se plantea hacer uso de un medio que permita mostrar esta cerámica en una plataforma virtual para difundir sus trabajos y que a la vez les permita tener contacto directo con el consumidor final. La propuesta se desarrolla considerando, en primer lugar, la identificación de los medios digitales utilizados por los miembros de la asociación, de modo que permita reconocer con qué plataformas cuenta esta comunidad. De esta forma, identifican que cuentan con una red social denominada Ichimay Wari Perú, en Facebook, administrada por el presidente de la asociación. En esta plataforma, se publican las diversas actividades en las que participan o que organiza la asociación, como eventos, ferias, actividades turísticas y los talleres dirigidos a estudiantes y turistas. No obstante, la frecuencia del uso en esta red social no es suficiente. 
Gracias a la identificación de los diversos aspectos, se presenta la propuesta del diseño de una plataforma virtual con el apoyo de un especialista a cargo del diseño de la plataforma. Los contenidos se refieren a la historia de la asociación, los productos para la venta, la importancia de la artesanía tradicional, la responsabilidad social y la sostenibilidad ambiental; también se considera que sea de fácil acceso y utilice un lenguaje sencillo.

Además, se propone el diseñó de un aplicativo móvil, para lo cual son necesarios algunos requisitos como contar con el ancho de la banda o cobertura del proveedor del servicio y que el aplicativo se descargue al dispositivo móvil. En este proceso de innovación los artesanos deben ser capacitados en el uso de la plataforma digital para que ellos mismos actualicen la información necesaria e incorporen los nuevos productos artesanales que elaboran.

\section{Referencias}

Bueno, A. (2003). Pachacamac: Informe de la expedición peruana William Pepper de 1896. Lima: Universidad Nacional Mayor de San Marcos. https://books.google.com.pe/books?id=HVWm8C79ciYC\&pg=PA81\&dq=pachacamac\&hl=es\&sa=X\&ved=0ahUKEwj__ Y3Lm8TiAhVMq1kKHWJeBp8Q6AEIJzAA\#v=onepage\&q=pachacamac\&f=false

Cáceres, M. (2019). Análisis del impacto de la producción masiva en la tradición ceramista de la comunidad Ichimay Wari, Lurín, 2018 [tesis de maestría, Universidad de San Martín de Porres]. Lima.

Hermoza Samanez, L. (2010). Buscando el perfil adecuado de proyectos de desarrollo desde el Arte y el Diseño para la comunidad de Huancarucma [tesis de maestría, Pontificia Universidad Católica del Perú]. Lima. http://tesis.pucp.edu.pe/repositorio/ handle/ 123456789/4784,

Hernández, R. Fernández, C. y Baptista, P. (2014). Metodología de la Investigación (6. a ed.). http://observatorio.epacartagena.gov.co/ wp-content/uploads/2017/08/metodologia-de-la-investigacion-sexta-edicion.compressed.pdf

Ministerio de Comercio Exterior y Turismo (MINCETUR). (s. f.). Artesanía Perú. https://www.mincetur.gob.pe/wp-content/uploads/ documentos/comercio_exterior/Sites/Pecex/lecturas_complementarias/otras_lecturas/Artesania_peruana.pdf

Navarro, S. (2015). La artesanía como industria cultural: Desafíos y oportunidades. Pontificia Universidad Javeriana, Bogotá D. C., Colombia. http://www.fessociologia.com/files/congress/12/papers/3519.pdf

Organización de las Naciones Unidas (ONU). (1992). Declaración de Rio sobre el Medioambiente y Desarrollo. http://www.unesco.org/ education/pdf/RIO_S.PDF

Organización de las Naciones Unidas (ONU). (2002). Informe de la Cumbre de Johannesburgo. https://unctad.org/es/ Docs/ aconf199d20_sp.pdf

Organización de las Naciones Unidas para la Educación, la Ciencia y la Cultura (UNESCO). (2003). Convención para la salvaguardia del patrimonio cultural inmaterial. https://ich.unesco.org/doc/src/01852-ES.pdf

Organización de las Naciones Unidas para la Educación, la Ciencia y la Cultura (UNESCO). (s. f.). Técnicas Ancestrales Tradicionales. https://ich.unesco.org/es/tecnicas-artesanales-tradicionales-00057

Zapata, V. A., Rojas, R. R. y Pereyra, C. N. (Eds.). (2013). Historia y cultura de Ayacucho. https://ebookcentral.proquest.com 
Milenka Cáceres Hurtado

Doctorando en Turismo; magister en Gestión Cultural Patrimonio y Turismo; licenciada en turismo y hotelería por la Universidad de San Martín de Porres. Docente universitaria en temas de patrimonio cultural, patrimonio y turismo.

mcaceresh@usmp.pe

ORCID: https://orcid.org/0000-0002-9999-5719

\section{Elizabeth Sara Gomez Castillo}

Magister en Ciencias Gastronómicas y licenciada en Ciencias de la Comunicación por la Universidad de San Martín de Porres. Docente e investigadora del Instituto de Investigación de la Escuela Profesional de Turismo y Hotelería de la Universidad de San Martín de Porres.

egomezc@usmp.pe

ORCID: https://orcid.org/0000-0001-6108-965X 